\section{THE SYSTEMATIC INVESTIGATION OF CHRONIC AFFECTIONS OF THE STOMACH AND INTESTINES.}

\section{BI GEORGE HERSCHELI, M.D. LOND.}

IT is a peculiarity of diseases of the stomach and intestines that from a study of the symptoms alone we are only able to divide them into groups the members of which, although apparently similar, depend upon widely differing conditions. The differentiation between these cannot in most instances be made by the ordinary methods of physical examination such as palpation, auscultation, and percussion. We shall require in most cases data which can only be acquired by other and more thorough methods of investigation.

It is doubtless true that we can often make a shrewd guess as to the real state of affairs by a careful study of the evolution of the disease and of the symptoms actually present; and this probability may be strengthened by the presence or absence of local tenderness, rigidity, tumour, or other signs which we may discover by a manual examination of the abdomen. But to make a real diagnosis with any degree of certainty we require additional information which can only be obtained by an examination of the stomach contents, stools, or urine.

It must, nevertheless, be understood very distinctly that it is not my intention to under-estimate the information to be derived from the anamnesis-i.e., the scientific questioning of the patient as to his previous history, the evolution of the disease, and the character of the symptoms. On the contrary, nothing can be of greater value as establishing the probability of the nature of the affection from which the patient is suffering. But we establish probabilities only, and the tentative conclusion at which we arrive requires to be confirmed by observed facts before it can aspire to the dignity of a diagnosis or definite opirion as to the nature of the disease. It is quite true that in cases of moderate severity and short duration we may be satisfied with such a tentative opinion, which may be confirmed by the happy result of treatment based upon the assumption. It is, unfortunately, also the fact that in daily practice such an assumption is frequently all that we are able to obtain, whether from the unwillingness or the inability of the patient to undergo a proper examination, and we have to do the best we can with it. But in all cases which are prolonged and resist treatment based upon the hypothesis which we have made, all cases accompanied by loss of flesh which cannot be accounted for by restriction of diet, all cases evidently progressive, and all gastric affections of any duration which occur in middle-aged persons of good previous health, it is our duty to impress upon the patient the absolute necessity for permitting a thorough investigation to be made. We must ever bear in mind that cancer of the stomach is the most frequent form of cancer, and that any dyspepsia which occurs in a man at the cancer age is very probably malignant. In fact, we may take it as a general working rule that any dyspepsia of severity accompanied by loss of appetite in a man between 40 and 60 is due to some serious organic lesion and that no sacrifice of time or convenience can be too great to have the matter settled before it is too late.

The aim of the present paper is to present to the physician who has acquired all the information possible from the anamnesis and the examination of the patient in his consulting room the additional data which modern methods enable him to obtain, and to critically discuss their value from a diagnostic standpoint.

We are able to ascertain the following facts: the length of time taken by the food to traverse the alimentary tract before it is expelled from the body; whether the fasting stomach is empty or contains substances which may afford us diagnostic information; to what extent digestion takes place in the stomach and how long food remains there; whether the pancreatic and biliary secretions are normal; whether there is evidence of chronic pancreatitis or of any affection of the bile-ducts; the presence of catarrh or abnormal putrefactive processes in the intestine; and whether there is bleeding into any part of the gastro-intestinal tract. These are the ordinary points upon which it is of ten necessary to be informed, and one or more of them may give us the additional data which we require to elucidate the case under consideration. Under conditions of especial difficulty we may not only have to put the patient through the whole series of tests but also to make use of the Roentgen ray, sigmoidoscope, gastroscope, examine the blood, or employ Salomon's test for malignant ulceration of the stomach.

I propose to discuss these points seriatim and to point out very briefly what we may learn from their investigations.

\section{A. - The Time taken by the Food to Traverse the Gastro- intestinal Canal.}

This may be estimated either by following the shadow of $\mathbf{a}$ bismuth meal upon the radiographic screen or by administering a dose of some inert colouring matter, such as carmine or vegetable charcoal, and observing when it appears in the stools. The radiographic method is unfortunately only at the disposal of those medical men who, living in large centres, have access to a properly equipped radiographic laboratory and to whose patients the question of expense is not important; or to the fewer number who have an installation in their house and the technical knowledge to use it efficiently. The charcoal or carmine capsule, besides being of universal application, will often reveal much more than the Roentgen rays, and must eventually be used in many cases.

Surprisingly little study has been accorded in this country by diagnosticians to the actual time taken by the food to pass through the alimentary canal, considering that its clinical importance is just as great as the daily number of stools. For instance, it is not at all unusual for a patient to have one stool a day and in consequence consider the bowels to be regular, whilst all the time there is a latent constipation with retention of fæcal matter, giving rise to chronic ill-health from auto-intoxication.

(a) In cases of diarrhoea the passage of the charcoal in the normal time would tend to show that the peristalsis in the small intestine was not increased and that the disturbance was in the lower portion of the large intestine.

(b) In diarrhoea the quick appearance of the charcoal or carmine in the stools would suggest that the trouble was in the small intestine or ascending colon.

(c) In a patient who has daily motions a dose of charcoal given at breakfast should appear in the next morning's stool. If it fails to do so there is latent constipation. If after the stool lavage of the sigmoid brings away black fæces there is retention in the lower bowel. If it does not do so the delay is in the cæcum, ascending, or transverse colon.

(d) In a patient who does not have a daily motion the removal of black fæces by lavage of the sigmoid on the morning following the administration of the cachet would show that atony or dilatation of the lower bowel was an important factor in the production of constipation.

(e) Appearance of black stools for several days after the administration of a dose of charcoal will point to atony of the bowel and latent constipation.

(f) By administering a dose of charcoal or carmine at the commencement of a test intestinal diet, we are able to mark off the moment when it commences to appear in the stools, and when we may safely use them for purposes of examination.

(g) We may mark off and obtain the total fæces corresponding to a test diet extending over a fixed period by giving a carmine cachet at its commencement and termination (provided that there is no latent constipation).

\section{B. - The Examination of the Fasting Stomach.}

This is especially useful as a first step in the investigation of cases in which there is a suspicion of serious disease. Not only shall we obtain valuable information as to the motor functions of the stomach, but even possibly special indications which the chemical examination of a test meal cannot afford.

With respect to the results obtained by this examination, we may divide our cases into four groups: the stomach may be empty ; it may contain neutral or alkaline contents; it may contain hydrochloric acid; or there may be present lactic or organic acids. Wach of the last three gronps may be again subdivided into those which contain food residues and those which do not.

1. The stomach is empty.-We are justified in arriving at the following deductions. (a) There is no considerable motor insufficiency of the stomach, as we know that the 
stomach is able to empty itself in 12 hours. In order to ascertain whether a lesser degree of loss of motility is present we must examine the stomach six hours after a mixed meal. We can thus practically exclude pyloric or duodenal stenosis. (b) TVe can exclude permanent hypersecretion of gastric juice, and thereby probably gastric duodenal ulcer. Consequently, any excess of HCI which we may find in our subsequent investigation must be alimentary - that is, limited to the digestive period. (c) We can probably exclude cancer of the stomach.

2. The fasting stomach contains a small quantity of neutral or alkaline fluid nith or nithout pus, blocd, or infusorix.-(a) A simple neutral or alkaline fluid containing epithelium and a few leucocytes would suggest achylia gastrica or chronic gastritis. (b) If pus and blood are present and if we can exclude phlegmonous gastritis (an affection of great rarity) and pus from the mouth, tonsils, post-nasal space, or bronchi there is in all probability an ulcerating cancer of the stomach. There must, of course, be an ulcerating surface to produce the pus or blood, and a non-malignant ulcer would hardly be found except in association with hydrocbloric acid. (o) The presence of infusoria in addition would make our diagnosis practically certain, and we should also be in a position to say that the cancer was situated upon the wall of the stomach and not at one of the orifices. Two varieties of protozoa are found in the stomach-the trichomonas hominis and the megastoma enterioum - when the four conditions are present which are necessary for their existence. These are absence of $\mathrm{HCl}$, an alkaline reaction, pouches or folds in the gastric mucosa, and absence of fermenting food residues. These conditions are only present in interostial carcinoma of the stomach.

3. The stomach contains hydrnohloric acid.-There may be no remnants of food or macroscopic or microscopic food residues.

(a) There are no food residues. If the hydrochloric acid is small in amount and only occasional the stomach is probably normal, as the secretion may be quite accidental and due to some temporary irritation or psychic excitation of the gastric glands. If the hydrochloric acid is invariably present or in larger amounts there is hypersecretion of gastric juice. Such a condition may be rarely a neurosis, or more probably due to ulcer of the duodenum or the irritation of gall-stones or of a diseased a ppendix.

(b) Hydrochloric acid with macroscopic food residues nearly always denotes an nlcer of the stomach or duodenum which has produced some amount of pyloric stenosis.

(c) Hydrochloric acid with microscopic food residues. Whenever hydrochloric acid is found in the fasting stomach the problem is to say whether we have to do with a simple hypersecretion of central or reflex origin or one due to the local irritation of retained food residues. The absence of macroscopic food residues is far from being conclusive that food is not retained abnormally in the stomach. Sarcinæ and yeast cells in chains or budding are in themselves strong presumptive evidence of food stagnation. If in addition we find muscle fibres, starch granules, fat globules, or crystals we can be certain that there are food residues retained in the folds and rugæ of the stomach, possibly slight in amount, yet sufficient to set up hypersecretion. Sarcinæ are practically never found in the stomach unless there is hydrochloric acid, and denote either benign stenosis of the pylorus or a carcinomatous ulcer in the early stage before the secondary gastritis has made much progress. As the atrophic changes develop in cases of cancer there will be observed a gradual diminution in the hydrochloric acid and sarcinæ with a proportionate appearance of the long bacillus and lactic acid. Consequently, if we find both degenerated sarcinæ together with the long bacillus we may deduce that the cancerous process is a rapid one. As sarcinæ require some time to grow their absence does not exclude stagnation of short duration.

4. We find food residues rith the presence of lactic acid and possibly the Oppler-Boas or long bacillus. - A practised observer can usually tell as soon as he looks through the microscope whether the gastric stagnation is associated with the presence of lactic or of hydrochloric acid respectively. In both cases you will see the same changes in the muscle fibres, in both you will of ten find yeast cells in chains, but if lactic acid be present the protoplasm of the leucocytes will be shrunken and granular, the cells still retaining a faint definite outline; if $\mathrm{HCl}$ is present the leucocytes will be entirely digested, leaving only centrally grouped nuclei. Also with lactic acid you may find the long bacillus, with $\mathrm{HCl}$ sarcinæ, and hardly ever the reverse.

It is important to bear in mind that the long bacillus is not diagnostic of malignant disease, but merely signifies food stagnation with absence of hydrochloric acid and presence of lactic acid. Recent work appears to point to the fact that the long bacillus is only suggestive of malignant disease when accompanied by a considerable amount of lactic acid. The reason is that whilst with ordinary albumins these bacilli form little acid, they find in the cancer albumins thrown off as the result of ulceration a medium which enables them to do "so in large quantities. It is obvious, therefore, that the long bacillus associated with large amounts of lactic acid will point to malignant disease which bas ulcerated. The long bacilli, which, according to Heineman and Hefferan, are identical with the Bulgarian bacillus, when present in large numbers frequently pass into the intestines and may be recovered from the stools by the same technique.

Macroscopic food residues show us that the stomach is unable to empty itself during the night and signify some obstruction in the pylorus or duodenum. The association of lactic acid points to its probably being of a malignant nature.

If the food residues are microscopic it is probable that we have a cancer infiltrating a small portion of the wall of the stomach. The immediate effect of this is a local loss of motility, which will increase pari passu with the extension of the affected area. At last a stage will be reached when the movements of that part of the stomach wall are not sufficient to dislodge the mucus with which it is covered, and food particles will lodge in the rugæ and on the surface.

\section{C.-Digestion in the Stomach.}

We should in all cases ascertain to the best of our ability the degree of gastric digestion, as even in affections apparently confined to the intestines the trouble may have a gastric origin. Indirectly we may infer an abnormality of gastric digestion when we find connective tissue remains in the stools, but the most satisfactory way of finding out what we require is by the direct examination of the stomach contents after a test meal.

The points which should receive our attention are:-

1. The presence of an abnormal amount of mucus. - This is a certain indication of chronic gastritis.

2. The $\mathrm{HCl}$ content of the gastrie juice.-(a) If normat. there may be respectively a normal stomach, simple atony of the stomach of insufficient severity to lead to retention of food residues, pyloric stenosis not sufficient to. delay materially the emptying of the stomach, or a pure neurosis. (b) Increase of $\mathrm{HCl}$ acidity. We may have nervous hyperchlorhydria, acid gastritis, a gastric or duodenal ulcer, a gall-stone, or a latent appendicitis. (c) Diminution of $\mathrm{HCl}$. This may result from a general condition, such as neurasthenia or anæmia, from a chronicgastritis, or from malignant disease of the stomach. (d) Absence of $\mathrm{HCl}$. This is always a most important symptom. Rarely a neurosis, it practically always denotes. either the terminal stage of a chronic gastritis or atrophy of the secreting glands either of benign or malignant origin.

3. Digestion of starch. - As a general rule the extent to. which starch digestion is carried out in the stomach may be taken as a confirmation of our findings as to the amount of free $\mathrm{HCl}$, being in inverse ratio to it. The chief practical use of ascertaining whether starch is digested in the stomach is to guide us in the selection of a suitable diet.

4. The secretion of the gastric ferments. - The estimation of the pepsin and rennin has at present very little value for diagnostic purposes, as they usually follow pretty closely the amount of $\mathrm{HCl}$ secretion.

A rough estimation as to the efficiency generally of proteid. digestion in the stomach may be made without passing a stomach tube by a simple examination of the stools. It appears that collagen, the basis of connective tissue, is not acted upon by the pancreatic juice unless it had been previously cooked or exposed to the action of $\mathrm{HCl}$. It follows therefore that if we administer to a patient connective tissue which is practically uncooked and find it reappear in the stools, we may be certain that there must be a defect in the

1 See author's work, Soured Milk and Pure Cultures of Lactic Acics Bacilli in the Treatment of Disease. London. 1910. 
amount of $\mathrm{HCl}$ secretion in the stomach. That is, of course, that we take care to give only such an amount as should in the ordinary way be efficiently dealt with by a healthy stomach. This is approximately the amount contained in 100 grammes of beef. If the beef is passed through a mincing machine, made into a cake and fried lightly on both sides in butter, so that the middle is still quite pink in colour, the connective tissue will be raw enough for our purpose.

\section{D.-Eridence of Functional Hepatic Insufficienoy and of} Disease of the Biliary Ducts.

It is probable that a pathological excess of urobilin in the urine always means either: (a) functional hepatic insufficiency ; $(b)$ some obstruction to the free flow of bile into the intestine; or $(c)$ auto-bæmolysis.

The chief clinical sign of hepatic insufficiency is diminution in the amount of urea excreted in the urine, in conjunction with increase in ammonia, uric acid, and purin bodies, and the presence in severe cases of leucin and tyrosin. But in order that the amount of urea may have any diagnostic significance it is necessary to make sure that it is not due simply to deficient ingestion of nitrogencontaining food. This could, of course, be done by estimating the nitrogen content of the diet of the patient, but in practice we secure the same results by the far simpler method of taking into account not the absolute amount of urea excreted in a day, but the relation between it and the total amount of urinary nitrogen. "Any lowering of the ratio of urea to urinary nitrogen below 83 per cent. is a sign of hepatic insufficiency.", 2

Interference with the free passage of bile into the intestine, such as is met with in chronic disease of the bile ducts or in gall-stone disease, is characterised by: (a) the same defective utilisation of fats as is met with in deficient secretion of the pancreas but to a lesser degree; (b) deficiency in the colouring matter in the fæces; and $(c)$ the presence in the urine of urobilin and bile pigment.

The presence of traces of bile in the urine is of greater diagnostic importance than is commonly supposed. Harry Adler ${ }^{3}$ has noted the fact that in many cases of obscure abdominal pain, in which gall-stones and chronic cholangitis were subsequently demonstrated by operation, he was struck by the fact that almost invariably traces of bile could be demonstrated in the urine by Rosin's test. (A green ring formed at the junction of the urine and a weak alcoholic solution of iodine.)

Cammidge and Robson ${ }^{4}$ state that bile pigment could be detected in the urine in 62 per cent. of cases of chronic pancreatitis associated with cholelithiasis and in only 16 per cent. of those in which no biliary calculi could be found in the common duct at the time of operation. Pathological excess of urobilin also appears, according to these observers, to denote some degree of obstruction to the free passage of bile into the intestine. The combination of both bile pigment and urobilin renders the existence of some degree of obstruction in the common duct practically certain.

One can distinguish between hæmatogenous and hepatogenous urobilinuria by the fact that in fresh urine urobilin is present, not as such but as a urobiligen, which oxidises into urobilin under the infuence of light. In urobilin derived from hæmolysis the reacticn will be obtained very soon after the urine has been passed, whilst in that resulting from the interference with the passage of bile into the intestine not until one or two days afterwards. We shall have, of course, in the former some degree of anæmia.

\section{E.-Defect in the Pancreatic Secretion.}

This can be ascertained from the examination of the stools after a test meal by finding :-

1. Undigested muscle fibre. -100 grammes of meat taken during the day by a healthy person should be entirely digested and absorbed, and no remnants, either macroscopic or microscopic, should be discoverable in the stools in an undigested condition. From numerous experiments it has been proved that muscls fibre is never completely digested in the stomach. The appearance of muscular fibres which have not lost their striation in the stools must point conclusively to

2 Combe: I'Autointoxication, p. 283.

\$ Medical Record, Oct. Cth, 1909.
The Pancreas : Its Surgery and Pathology. London. 1907. defect in the proteid digestion in the intestine, and necessarily implies that the pancreatic secretion is deficient.

2. Alteration in the utilisation of fat.-(a) Excess of saponified over unsaponified fat in the fæces may point to increased activity of the pancreatic secretion, and is frequently met with in hyperchlorhydria as the direct result of the excess of free $\mathrm{HCl}$, the natural activator of the pancreas. Such an excess of pancreatic secretion is, I am sure, exceedingly common as the pancreatic catarrh which forms the first stage of chronic pancreatitis. Excess of saponified fat may also be due to the action of bacteria, and points to abnormal putrefactive processes in the upper part of the intestine. When this is the case there will be invariably other evidences of putrefactive processes in the intestine, such as indicanuria, and the usual syndrome of autointoxication. There will also naturally be evidence of defective pancreatic secretion, such as the presence of undigested muscular fibre and the positive result of the tryptic insufficiency test, and we shall, under these circumstances, be justified in assuming that the excess of saponified fat means intestinal catarrh.

(b) Diminution of saponified fats. This will point to some degenerative change of the pancreas, is of ten met with in the later chronic stage of intestinal indigestion, and in conjunction with the pancreatic $\mathrm{C}$ reaction will suggest cirrhotic changes in the pancreas due to long-standing chronic inflammation.

(c) Excess of unabsorbed fat. This will show that there is some interference with the functions of the pancreas. provided that we can exclude defective gastric digestion, biliary obstruction, and an excess of fats in the food, especially those, such as mutton fat, having a high melting point.

3. Iryptic insuficuency test. - Of all the methods which have been devised for measuring the functional activity of the pancreas, the most certain and direct is that which consists of determining the presence of trypsin in the fæces. It is a well-known fact that a certain proportion of the trypsin of the pancreatic juice is not used up in the duodenum, and may be found in the stools. Nature is not parsimonious, and habitually supplies an excess of digestive fluids over the amount actually required. It follows, therefore, that if we can demonstrate the presence of trypsin in the fæces there must be enough and to spare of pancreatic juice secreted, and there can be nothing very wrong with the pancreas in the direction of functional failure. If we find a relative excess of trypsin in the fæces we have strongly suggested a condition of catarrhal hypersecretion of the pancreas, such as is met with in the first stage of chronic pancreatitis. If, on the other hand, we find diminution or absence of the tryptic ferment in the stools, we may assume that there is some affection of that organ which materially interferes with its secretion.

The best method at present at our disposal for estimating the amount of trypsin presert in the stools appears to be that devised by Gross, ${ }^{5}$ which is based upon the time taken by an aqueous extract of a given quantity of fæces to digest a standard weight of casein. To secure the best results it is important that the patient should be at the time upon a dieto consisting mainly of proteids, this having been shown to produce the largest output of trypsin in a healthy person. Normally, 10 cubic centimetres of a 1 in 3 suspension of fæces should digest three-quarters of a grain of casein ir from 12 to 14 hours. In abnormal conditions the time may be much reduced or extended. One ascertains the moment wher all the casein is digested by failure to obtain a precipitate with acetic acid.

\section{F.-Evidence of Chronic Pancreatitis.}

It is now quite certain that chronic pancreatitis is a mach commoner affection than has hitherto been imagined, and that it explains the pathology of a certain proportion of obscure gastro-intestinal disturbances. In many cases of chronic pancreatitis, especially when resulting from infective extension from a duodenal catarrh, there may be no pain or jaundice, but merely a dyspepsia of the atonic type with flatulence as a prominent symptom. It is consequently necessary to definitely exclude pancreatitis before assuming the presence of an uncomplicated gastric catarrh, it being morally certain that many cases of so-called gastric catarrh remain uncured by treatment because they are in realit $\$$ chronic pancreatitis. 
Again, as I have pointed ont elsewhere, ${ }^{\circ}$ the presence of chronic pancreatitis probably has considerable diagnostic value in the recognition of duodenal ulcer, as if gall-stones can be excluded a local source of irritation in the duodenum is suggested. This, in the presence of rigidity of the recti muscles, hunger pain, or abdominal tenderness, may give the casting vote against a hyperchlorhydria of functional origin.

For the recognition of chronic pancreatitis we rely mainly upon the Cammidge $\mathrm{C}$ reaction ${ }^{\tau}$ in conjunction with the other signs of abnormality in the pancreatic juice already mentioned. According to Cammidge and Robson, the presence of crystals of oxalate of calcium in the urine are strongly contirmatory of chronic pancreatitis, having been found in 73 per cent. of all cases examined. They do not appear to occur in acute pancreatitis, and very rarely in malignant disease of that organ.

\section{G.-Signs of Abnormal, Putrefactive Processes in the Intestine.}

We cannot prescribe intelligently for any case of neurasthenia, intestinal dyspepsia, or diarrhoea unless we know whether or not there are abnormal putrefactive processes in the intestine.

The signs upon which we found our opinion are urinary (excess of indican, excess of conjugate sulphates, and the presence of phenols and their congeners) and fecal (the excess of Gram-positive over Gram-negative forms in stained cover-glass preparations, alkalinity of reaction, excess of anaerobic bacteria, and abnormal fermentation in Strasburger's tubes).

\section{H.-Signs of Intestinal Catarrh.}

The diagnosis of intestinal catarrh is made chiefly from the demonstration of mucus and an increased amount of inorganic ash in the fæces.

Mucus in the fæces must always be considered as pathological, and to denote either a functional disturbance of secretion or more frequently an anatomical disease. We are enabled to deduce from the appearance of the mucus and the way in which it is mixed with the fæcal matter the part of the intestine from which it comes. Pure mucus without any admixture of fæces denotes catarrh of the rectus, sigmoid, or lowest part of the descending colon. Fæcal masses covered exteriorly by a thin layer of mucus point to constipation, fæcal retention, and irritation, and consequent catarrh of the lower bowel. Mucus, whether macroscopic or microscopic, if intimately mixed with fæces, suggests catarrh of the upper colon or of the small intestine. If the flakes of mucus are either bile-stained or become green when acted upon by a strong aqueous solution of corrosive sublimate, it may be considered practically certain that they come from the small intestine.

The amount of inorganic ash in the fæces varies normally between 10 and 15 per cent. It is increased in catarrhal inflammation of the intestine, and this increase is greater the nearer the lesion is to the anus. A large or considerable excess of inorganic ash renders the diagnosis of chronic colitis practically certain and is a valuable confirmation of other signs.

\section{I.-Evidence of Bleeding into Any Part of the Gastro- intestinal Canal.}

One of the most marked features of the last two or three years has been the improvement in the technique for detecting minute amounts of blood in the fæces (occult blood), and our tests are now quite delicate enough for all practical purposes. As regards the significance of the finding, observers are mostly agreed that a negative result of the test is much more valuable from a diagnostic point of view than a positive one. For instance, in the absence of hydrochloric acid in the stomach contents with no retention of food, the continued absence of occult blood in the stools would go far to negative the suggestion of a gastric carcinoma.

The information to be obtained from the presence of occult blood in the stools, the patient being on a hæmoglobin-free diet, may be briefly stated as follows:-1. The constant presence of occult blood strongly suggests malignant

6 The Diagnosis of Duodenal Ulcer, with Special Reference to the Pancreatic Reaction, Clinical Journal, Jan. 12t b, 1910

The Pancreas, its Surgery and Pathology, by Mayo Robson and Cammidge, London, 1907, p. 252 . disease of the stomach or intestines. 2. Intermittent presence suggests a gastric or duodenal ulcer. 3. Appearance of occult blood in the stools of a patient who has been apparently cured of an ulcer of the stomach or duodenum strongly points to an impending relapse. As Boas has wisely suggested, all cases of cured ulcer should have periodical examinations of the stools for occult blood for some months until the continued absence of blood shows complete healing. 4. The test for occult blood is of extreme value to us during the medicinal treatment of stomach or duodenum, to tell us when it is safe to make any improvement of the diet. Should occult blood appear at any time after an increase of food has been given the patient it wil show that the change has been prematurely made and we must try back for a little.

In any case the appearance of occult blood in the stools points out that there is some bleeding going on in the gastrointestinal tract, and indicates the absolute necessity for a thorough examination and investigation of the patient

I hope that by this time it is quite plain, first of all, that a real diagnosis of a case of disturbance of the stomach or intestines can only be made by a study extending over several days, and that an opinion arrived at in any other manner must be a pure guess, possibly correct, but still only a guess. Secondly, that there is no need to despair if, after having asked his patient the usual questions and palpated the abdomen, one is not able to say definitely anything more than that the patient has "indigestion." I hope that I have succeeded in showing that there are still methods to be tried before the physician is justified in treating the case by the method of trial and error or sending it to the surgeon. It only remains to consider the best method of putting the patient through the different tests.

The first difficulty is usually with the patient himself. $\mathrm{He}$ would be perfectly satisfied with a diagnosis such as " acid dyspepsia," and it is difficult to make him understand that it is not a diagnosis at all, but simply a statement in other words of what he knows already. And he naturally cannot understand why, if a diagnosis of a disease of heart or lungs can be made at once in the consulting-room, those of the digestive system should require further investigation and be in a different category. His natural tendency is to lose confidence and to go to someone else who will diagnose "acid dyspepsia" or " constipation" or something similarly meaningless, and not put him to the trouble or expense of further investigation.

But assuming that the patient really wishes to get well and is willing to take some trouble to do so, how shall the tests best be carried out?

The very first thing is to decide which tests are necessary in the particular case under investigation, as it may not be necessary to go through the formidable list which $I$ have enumerated. In many cases there may be just one or two things which we require to ascertain to clench the diagnosis and it frequently happens that it is not necessary to pass tube into the stomach of the patient, as we may obtain all the additional data which we must have from the examina tion of the stools and urine. In any case it is as well to commence the investigation by a thorough analysis of these, as we cannot fail to acquire an immense amount of definite information as to the condition of the gastric and duodenal digestions, the condition of the pancreas, and the presence or absence of intestinal catarrh, information which will safely guide us as to the extent and nature of the further investiga. tions required for the elucidation of the case.

The next point is to make arrangements for obtaining the facilities necessary for the efficient performance of the tests. Even in a case in which the investigation is limited to the examination of the stools and urine we shall require : $(a)$ the collection and measurement of the 24 hours urine; $(b)$ the daily collection of the fæces, and opportunities of inspecting and microscopically examining them in a fresh condition, separating the carmine-coloured portions ; $(c)$ a period during which the patient is upon a test diet containing measured amounts of meat, bread, milk, potatoes, \&c. ; and $(d)$ a second period during which all hæmoglobin is excluded from the patient's diet in order that the stools may be examined for occult blood.

To attempt such an investigation in the patient's home is almost impossible; it would necessitate a very large amount of detailed instruction on the part of the medical man, as the work would have to be carried out either by the untrained 
friends of the patient or by hired nurses who would be probably equally unversed in the minutiæ of the diets and methods. The preparation of the test diets not only requires scales and weights, but necessitates an alteration in the usual household arrangements, and throws a certain amount of extra work upon often unwilling domestics. Besides, the medical attendant will have to pay visits at unusual and inconvenient hours, such as before breakfast. The collection of stools and urine is not by any means a task which will be undertaken with alacrity by ordinary servants.

Taking all these facts into consideration, it is far better for the patient to enter a nursing home for a week, into which space of time the necessary tests can usually be compressed. Here the preparation of the different test meals, the administration of the enemas, the collection and preservation of the stools, are part of the daily routine and are carried out as a matter of course, and the medical man can visit early or late without apsetting or inconveniencing anybody.

Harley-street, W.

\section{MATERNITY CASES IN THE LIVERPOOL WORKHOUSE HOSPITAL DURING 1907, 1908, AND 1909.}

BY WILLIAM ALEXANDER, M.D. R.U.I., F.R.C.S. ENG., VISITING SURGEON TO THE INSTINUTION.

IN The LANCET of April 6th, 1907, p. 940, there is a report upon the maternity wards of the Liverpool Workhouse Hospital for 1906. The present communication is a similar report for the years 1907, 1908, and 1909 .

Total number of women admitted, 1330; married, 761 ; single, 569 ; primiparæ, 455 . Residing in the workhouse for variable times before confinement, 382 ; admitted direct from town when in labour, 926 ; admitted from the workhouse lock ward, 22

Of maternal mortality there were six cases, details of each of which are here given in condensed form.

1. Case 163, 1909.-Aged 40; 12-para; admitted in labour suffering from vomiting and albuminuria. Confinement natural. Baby premature and stillborn. Vomiting persistent after labour. Suppression of urine set in gradually and continued until death, six days after. Post-mortem examination : Kidneys much congested and large, pelvis normal, increase of fluid in pericardium, and general odema.

2. Case 350, 1909.-Age 36; 5-para. Normal labour Sept. 15th. Albumin in urine. Chest tubercular. Patient sent to the medical wards, where she died from uncomplicated phthisis on Oct. 9th. No post-mortem examination allowed.

3. Case $90,1908 .-$ Age 29; 4-para. Admitted with a temperature of 101.8\% F. Pleuro-pneumonia diagnosed. Double pneumonia supervened

and the patient died seven days after from that disease.

4. Case 172, 1907.-Age 21. Patient admitted from the medical wards in a state of collapse, with constant vomiting for three days previous to admission. She did not rally, but died next day from sys previous to admission. She did not rally, but died next day from sy

signs of cedema of the lungs. Po post-mortem examination. food for ten days before admission. Powerless labour, forceps applied. Died from sepsis. Abscess found in broad ligaments, pus in uterus, probably previous pyosalpinx.

6. Case 394, 1907. - Age 22. Labour quite normal. Seven hours after6. Case 394, 1907 - Age 22 . Labour quite normal. Seven hours after-
wards eclampsia set in and patient died in 28 hours, no remedies having
the slightest effect on the course of the disease. A necropsy showed the slightest effect on the course of the dise
oedema of the lungs and cerebral congestion.

The above are all the deaths from every oause of every noman who was admitted to the maternity wards during these years, and inolude all who have died from any cause during the month followng their confinement. The statement in the Hospital of Jan. 15th, 1910, p. 466, that we only count deaths from purely maternity causes is absolutely false, and we shall be only too glad to show our books to anyone interested in the truth of the matter.

Infantile mortality.-Mature, dying soon after birth, 22 ; mature, stillborn, 15 ; premature and immature, 78 ; premature and decomposed, 82 . Of the mature the causes of death were debility, cyanosis, syphilis, and apnoea, but in most of the cases debility was the cause. Of the mothers admitted during 190919 had suffered, or were suffering, from syphilis. In 6 cases syphilis was suspected, but the grounds for suspicion were not conclusive. In the other years the proportion of syphilitic children has not been made out, but 1.s probably much the same.

Children born.-Males, 704 ; females, 653 ; twins, 27.

Presentations.-Head, 1272 ; transverse, i1 ; breech, 46 ; knee, 1 : foot, 23 ; face, 4 ; shoulder, 1 ; with cord, 17. Operations. - Furceps, 57 : versions (podalic), 14.
Complications.-Eclampsia, 3 ; albuminuria, 32 ; pneumonia, 4 ; hæmorrhage, 45 (accidental 26 , unavoidable 15 , post-partum 4); adherent placenta, 4 ; adherent membranes, 5 ; retained placenta, 1 ; ophthalmia in infants, 13 (neonatorum 4, catarrhal 9).

Number of mothers removed from the maternity wards on account of temperature, 5.

Number of midwives trained during the three years, 61. Of these 37 entered for the Central Midwives Board examination, two of whom failed. 25 nurses also had each a month's training in monthly nursing.

\section{Remarks.}

The maternal mortality is 0.45 per cent., a fractional quantity of which we are very proud considering the class of mothers we have to deal with, as indicated by the large number of immature babies who survived birth by only a few hours, and the still larger number of dead and decomposed infants that are to be found in the statistics. This lying-in hospital has been in constant use, and our records date back to 1858 , more than half a century ago. It has been celebrated all that time for its remarkably low maternal mortality. In her notes on lying-in institutions published in 1871 Miss Nightingale refers in some detail to the statistics of the Liverpool Workhouse Maternity supplied to her by my predecessor, Mr. Barnes, and she gives in a table the admissions and the maternal mortality for the five years 1866 , $1867,1868,1869$, and 1870. The admissions were 2466, and the maternal mortality 13 , or 0.53 per cent., a very much more remarkably low mortality at that period than is the 0.45 per cent. mortality for the past three years. The term asepsis was then unknown, and the architectural arrangements were the same except that the lavatories were more awkwardly situated than they are now. Yet the results were the envy of maternity hospitals throughout the country, and many deputations visited us to ascertain the conditions that brought us such safety. Many suggestions were made by these visitors as to the supposed cause; most were, however, unwilling to recognise that our charter of safety lay in our straw beds, beds that were discarded as soon as used and of which each patient used three, one in the delivery ward, one in the lying-in ward, and a third in the recovery ward, as described in THE LANCET in the article dealing with the same wards for 1906. They went away in sorrow because they had large possessions of valuable mattresses (hair), feather pillows, \&c., and they could not stoop so low as to adopt straw beds. Fortunately for them, antiseptics and asepsis have allowed full scope for both safety and expense, and our workhouse maternity has now lost its pre-eminence in safety, but maintains its equality in that direction, while its pre-eminence in economy is much more marked than it ever was before. We have given up the straw beds owing to the introduction of fibre, which makes a cheap, easily replaced, and a more comfortable bed, and flock pillows have been substituted for straw ones.

Much the same routine is followed as was described in THE LANCET, but each patient now has only two beds during her passage through the lying-in, the delivery bed being removed from the maternity ward when the mother passes into the recovery ward. Here she obtains a new bed which has never been occupied by any person before, and this she uses until she leaves the maternity division. The bed is then sent away, never to return. The delivery bed is likely to become soiled with discharges, \&c., and hence it is only used during delivery. From the behaviour of the patients it may be concluded that the fibre beds are very comfor table; the sleep is sound, and no complaints have ever been made about their hardness.

In the Hospital of Jan. 1st, 1910, p. £95, Sir Henry Burdett says : "We may point out although classification is essential for moral reasons in the general wards of a Poor-law infirmary, in the maternity wards it is doubly necessary on moral and hygienic grounds. No board of guardians has the right to subject any inmate of a Poor-law infirmary in any department to the risks of contrasting a loathsome contagious disease, much less to permit infants to be subjected to such risks." In another place he sars: "There they will see girls of 16 and young women who are physically bealthy crowded together in the same wards with older wcmen who may be suffering from contagious distases of the most repulsive kind."

Now this picture is calculated to stir up the emotions of tender-heatted $m \in n$ and women, but it is drawn from the 